\title{
Mujeres con competencias STEM en el área de metal mecánica
}

\section{Women with STEM skills in the mechanical metal}

\author{
JIMÉNEZ-HIDALGO, Guadalupe "“†*, MANCERA-VALENCIA, Federico Julián" y SÁNCHEZ \\ LUJÁN, Bertha Ivonne
}

'Tecnológico Nacional de México campus Ciudad Jiménez. Av. Tecnológico S/N (1.018,75 km) 33980 Ciudad Jiménez, Chihuahua, México.

"Centro de Investigación y Docencia (CID). Lucio Cabañas 27, Lomas del Santuario Etapa II, Pablo Gómez, 31206 Chihuahua, Chihuahua, México.

ID $1^{\mathrm{er}}$ Autor: Guadalupe, Jiménez-Hidalgo / ORC ID: 0000-0002-8257-3093, CVU CONACYT ID: 1013757

ID $1^{\mathrm{er}}$ Coautor: Federico Julián, Mancera-Valencia / ORC ID: 0000-0003-3467-3441, CVU CONACYT ID: 214503

ID $2^{\text {do }}$ Coautor: Bertha Ivonne, Sánchez-Luján / ORC ID: 0000-0002-3595-8281, CVU CONACYT ID: 342583

DOI: $10.35429 /$ JET.2019.9.3.24.29

Recibido: 01 de Julio, 2019; Aceptado 22 de Septiembre, 2019

\begin{abstract}
Resumen
La inserción a la educación superior de las mujeres no ha sido una tarea fácil desde la apertura de las universidades, quedaron fuera de este beneficio, logrando en 1887 el examen profesional de la primera Doctora de la Facultad de Medicina, aun en la actualidad existe carreras donde la presencia de ellas es reducida como es el caso de Ingeniería Mecatrónica e Ingeniería Electromecánica en el Tecnológico Nacional de México campus Jiménez. Las habilidades en Ciencia, Tecnología, Ingeniería y Matemáticas son un factor clave para inserción de las mujeres en estas licenciaturas, se presenta el avance de investigación narrativa de lo que ha favorecido su elección de profesión dentro del proyecto "Participación de las mujeres en la inserción de las carreras con matricula predominantemente de hombres", el análisis de 14 estudiantes mujeres del TecNM campus Jiménez, nos muestra como las competencias STEM están presentes en sus decisiones para optar por su futuro laboral.
\end{abstract}

Mujeres, Matemáticas, Educación superior

\begin{abstract}
The opening to the higher education of women has not been an easy task since the existence of the universities, they were left out of this benefit, in 1887 the professional examination of the first Doctor of the Faculty of Medicine, even today there are careers where their presence is reduced as is the case of Mechatronics Engineering and Electromechanical Engineering at the Tecnológico Nacional de México, campus Jiménez, the skills in Science, Technology, Engineering and Mathematics are a key factor for the insertion of women in these degrees, presents the progress of narrative research that has favored their choice of profession within the project "Participation of women in the insertion of predominantly men's degree", the analysis of 14 women of the TecNM campus Jiménez, shows us how the STEM competencies are present in their decisions to opt for their future job.
\end{abstract}

Mujeres, Matemáticas, Educación superior

Citación: JIMÉNEZ-HIDALGO, Guadalupe, MANCERA-VALENCIA, Federico Julián y SÁNCHEZ LUJÁN, Bertha Ivonne. Mujeres con competencias STEM en el área de metal mecánica. Revista Teoría Educativa. 2019. 3-9: 24-29.

\footnotetext{
* Correspondencia del Autor (correo electrónico: gpejimenezh@yahoo.com)

$\dagger$ Investigador contribuyendo como primer autor.
} 


\section{Introducción}

Las mujeres en la educación fueron excluidas, en un principio las universidades aceptaban solamente hombres. La universidad Alemana autoriza el ingreso de Anna María Von Shuurman, con la condición de permanecer en un cuarto de madera colocado dentro de la misma aula universitaria, separado por una pared en la que se habían practicado agujeros, evitando el contacto con los estudiantes y docentes. Greña (2008).

La educación es un factor relevante en el desarrollo de los países. La educación es un derecho de todos, de hombres y de mujeres. (Solihati, 2019).

El acceso de las mujeres a la educación superior implicó obstáculos de diversa índole, dependiendo de la legislación de cada país. En su mayoría las primeras universitarias optaron por carreras relacionadas con las Ciencias de la Salud y específicamente Medicina. Provocaron y participaron de un debate en la sociedad, relacionado con la capacidad de las mujeres para acceder a los estudios universitarios. (Palermo, 2006).

La formación en áreas del conocimiento de ciencia, tecnología, ingeniería y matemáticas o STEM (acrónimo en inglés de science, technology, engineering y mathematics) hace referencia a la tendencia educativa a formar a futuros profesionistas a partir de cuatro disciplinas: ciencia, tecnología, ingeniería y matemáticas, con un enfoque interdisciplinario, práctico y aplicado (Arredondo, 2019).

En su mayoría los programas o proyectos que intentan fomentar las competencias en las áreas de STEM tienen la motivación de atraer a estudiantes de poblaciones vulnerables o insuficientemente representadas, ya que se considera que estas habilidades pueden significar un factor relevante para su inclusión económica. Se considera a las mujeres uno de estos sectores, que, según datos acerca de la eficiencia terminal de las universidades, son significativamente menos propensas a seguir una carrera universitaria o estudios de especialización enfocados en campos de STEM (Million Women Mentors, 2015).
La falta de inversión en el desarrollo de competencias en STEM ya no queda únicamente como una carencia educativa que puede sobrellevarse con la capacitación para el empleo, sino refleja la manera en que los países podrán hacer frente a las necesidades laborales del futuro, la posible proyección sobre sus industrias y la competitividad que tendrán ante el resto de las naciones.

El Tecnológico Nacional de México (TecNM) es un organismo dedicada a la educación superior tecnológica que está constituido por 254 instituciones, de las cuales 126 son Institutos Tecnológicos federales, 128 Institutos Tecnológicos descentralizados en todo el territorio nacional incluida la ciudad de México. (TecNM, 2019).

El TecNM campus Jiménez ofrece cinco carreras profesionales en ingenierías: Ingeniería Industrial, Ingeniería Electromecánica, Ingeniería en Sistemas Computacionales, Ingeniería Mecatrónica, e Ingeniería en Gestión Empresarial, además de otra licenciatura: Contador Público, dentro de esta diversidad de profesiones, se detectó que la menor participación de las mujeres se encontraba en el campo de electromecánica y de mecatrónica, lo que impulso a realizar una investigación sobre la inserción de las mujeres en estas licenciaturas en particular.

\section{Desarrollo}

Al hacer una análisis de la matrícula de las carreras en la institución, se observan 14 mujeres estudiando en el campo del metal mecánica, dentro de los semestres octavo, sextos, cuarto y segundos de su retícula universitaria, debido a que la institución solo cuenta con ingresos en el periodo Agosto - Diciembre de cada año.

Para el área de metal mecánica las competencias STEM son una base importante en el desarrollo curricular de sus profesiones.

Las mujeres que van dejando huella en el camino de la ingeniería presentan una inclinación por las matemáticas desde temprana edad, son personas que reconocen sus potencialidades, sus limitaciones y rompen los esquemas impuestos a ellas. 
Participan activamente en el mostrar sus capacidades, Atkinson (2005) nos hace reconocer que las experiencias, las memorias, las emociones y sensaciones individuales son generadas por una formación del colectivo culturan donde se contextualiza, por lo que los objetos, las imágenes, las evocaciones son elementos relevantes de análisis para comprender de otra forma las realidades. Por lo que se procedió a realizar entrevistas a cada una de ellas, a recopilar su información académica, y documentación que permitiera reconocer sus competencias, para realizar un análisis de la información.

\section{Metodología a desarrollar}

En el contexto de rescatar las percepciones de las alumnas de educación superior del Tecnológico Nacional de México campus Jiménez respecto a su rol de estudiantes generando competencias en ciencias, tecnología, ingeniería y matemáticas (STEM por sus siglas en inglés), es investigar sobre la cotidianidad escolar y personal de las mujeres a nivel universitario, para lo cual se recurrió a un estudio cualitativo debido a que se indaga sobre su realidad de lo que permite en su contexto las competencias STEM.

Comprender los significados que tienen para las estudiantes en grupos mayoritariamente de hombres, su participación activa, por lo que se optó por realizar entrevistas semiestructuradas a 14 mujeres estudiantes de las carreras de Ingeniería Electromecánica e Ingeniería Mecatrónica. Se planteó mantener una conversación con las informantes "para entender el mundo desde la perspectiva de los entrevistados y desmenuzar los significados de sus experiencias" (Álvarez-Gayou, 2003).

La investigación narrativa, cuenta con sujetos que la van conformando, él informante, él intérprete, y él lector auxiliado de los textos que concentran lo que se narra y el informe que se crea. La recolección de los datos son recreados en narrativas que generen análisis, que den sentido a la experiencia desarrollada por el informante, que permita observar con una mirada crítica la escena.

Es hermenéutica porque es un traductor de texto, comunica tal como los interpreto, hago mi propia traducción del discurso y genero una nueva narrativa.
Existen tradicionalmente dos enfoques de análisis (Bold, 2011) un enfoque estructuralista que se centra en la búsqueda de elementos comunes dentro de los relatos e historias, examinando su estructura más que su significado. Una segunda vía (enfoque temático), se centra en el significado dentro de las narraciones (Bolívar, 2012).

Se utiliza un análisis temático, debido a que se busca en el conjunto de narrativas de las estudiantes de ingeniería sus características, que las llevan a ser parte del fenómeno del cambio.

Se designaron claves para dar cuenta de las versiones vertidas por las entrevistas realizadas estudiantes mujeres, docentes y se dio una codificación que indica a que grupo pertenece, una letra para identificar a la persona, semestre al que pertenece, y carrera en que se desenvuelve.

La investigación narrativa permite dar cuenta de aspectos que son relevantes, como los sentimientos, propósitos y deseos que con otras formas de investigación quedarían fuera (Huchim, 2013).

\section{Resultados}

A partir de las entrevistas podemos iniciar a indagar sobre las competencias STEM que presentan las estudiantes, la primera competencia identificada es la de matemáticas, donde el grupo de mujeres considera que es una de sus fortalezas como nos dicen:

"La primaria donde estuve fue muy humilde, había muy pocos alumnos, es de un ranchito, siempre me destaque por tener muy buenas calificaciones, de hecho siempre iba a concursar, de lo que me acuerdo, gané una medalla, en matemáticas tengo varios reconocimientos. Tuve un año el mejor puntaje del estado, creo en tercero de secundaria, pero en matemáticas nada más". ES 8IM

"En la primaria era muy inteligente estuve en muchos concursos de conocimiento". EA 8IMA

"Yo estuve en la primaria en todas las actividades que se pudiera deportivas, culturales, académicas en todo, mi profesor me decía oye: va a haber este concurso para que te metas, ¡vamos a prepararte para oratoria!. 
Una habilidad que tengo mucho es la numérica me gustan mucho todas las operaciones matemáticas, este las resuelvo algunas mentalmente, cuando veo algún problema ya me imagino más o menos como solucionarlo". EB 6IM

“Gane el premio académico en segundo año de primaria, pero ya después como que no me enfoque en ganar premios y ya solamente disfrute la primaria pero ya nunca baje el promedio de nueve en la primaria y también era de las que más participaba, porque era muy seria, pero participaba; en la secundaria ahí si me concentre mucho en mi promedio y tampoco lo baje de nueve". EN 6IM

No sólo se trata de las percepciones de las estudiantes, sus calificaciones en matemáticas en su primer semestre les permite corroborar su competencia. Se verificaron sus calificaciones por medio de sus Kardex que les son otorgados por servicios escolares de la institución, validando su información.

“Aprobé con una calificación de cálculo diferencial 97”. ES 8IM

"El cálculo se me dificultó, obtuve un 85". EA 8IM

"La calificación de cálculo diferencial fue de 100". EB 6IM

"Mi calificación de cálculo en el primer semestre fue de 97”. EL 6IM

Como se observa el desarrollo de su potencial en matemáticas se fue desarrollando desde la educación básica en su mayoría, es una inteligencia que se puede favorecer con trabajo continuo, el concepto de inteligencia ha cambiado a través del tiempo, y de acuerdo a las distintas sociedades existe un ideal de ser inteligente.

Las estudiantes se perciben con una inteligencia Lógico-Matemática: Tiene que ver con una confrontación de objetos, en su ordenación y reordenación y en la evaluación de su cantidad, lo que lleva a un desarrollo de pensamiento abstracto, con la precisión y la organización a través de pautas o secuencias.
Comprende la capacidad para manejar operaciones matemáticas y razonar correctamente, realizar planteamientos aún con la ausencia de los objetos. Se acercan a los cálculos numéricos, estadísticas y presupuestos con entusiasmo. Se utiliza para resolver problemas de lógica y matemáticas (Sánchez, 2016). Para estas mujeres la tecnología es un elemento importante en su vida, sus percepciones en la elección de carrera van encaminadas a las TIC.

"En bachillerato decidí estudiar la carrera de programación, porque siempre me ha llamado mucho la atención la computación y programar, por eso entré a esa especialidad, me acuerdo que nos llevaron a el tecnológico de Monterrey estaba la carrera de ingeniería en mecatrónica, entonces a mí me llamo mucho la atención, cuando dijeron que los alumnos hacían innovaciones en las prótesis y después las regalaban a personas necesitadas, ¡tenía que entrar a mecatrónica y luego a una carrera de biología!". ES 4IM

"Pienso que sí tengo habilidades para programar, me he dado cuenta que me sale más o menos la lógica para hacerlo". EL 6IM

"En el bachillerato estudié electromecánica, al principio yo quería estudiar algo de alimentos porque quería seguir el ejemplo de mis papás, quería ser doctora pero me tocó en la tarde, yo quería cambiarme en la mañana y solamente había espacio en electromecánica, me gustó la carrera y aparte desde pequeña siempre andaba con mi abuelito que tenía un taller, una vulca y me gustaba ahí andar de manillenta con él. Empecé adentrarme más a lo que era los circuitos eléctricos o la programación y fuimos a participar a prototipos y obtuvimos el primer lugar en la modalidad tecnológica, era como que la más codiciada en ese evento y pues la verdad se sintió muy bien". EC 2IM

"Siempre me ha llamado mucho así por ejemplo arreglar lo celulares o saber a qué le mueven o que hay dentro y pues dije una carrera que sea más o menos que sea de eso o de máquinas, no se moverle y pues ya me dijeron que era una de las dos electromecánica o mecatrónica. 
Me empezó a gustar mucho lo de las máquinas, la electricidad me llamo mucho la atención, elegí electromecánica". ES 2IE El iniciar a desarrollar las competencias en matemáticas y tecnología a temprana edad facilita el camino, sin limitar la oportunidad a quien opta por una carrera ingenieril, iniciando con su proceso de asimilación de la tecnología.

“Al estudiar allí en el rancho pues nada de tecnología también en eso me fui un poco más abajo porque yo llegando acá a Jiménez ya fue como que ;Ah la computadora;, porque en secundaria y en primaria no vi nada, noooo tuve nunca una computadora, la computadora la tuve hasta que entre a aquí al Tec mi hermano fue el único que la tuvo pero también hasta que entro al Tec, entonces no tuve mucho contacto con tecnología y en eso también me vi un poco en desventaja cuando llegue aquí, ¡mmmhh! pero creo que, que las bases sí estuvieron bien, porque si me pude ya poner al corriente con lo que ya venían un poquito más avanzados". ES 8IM

$\mathrm{Al}$ elegir su carrera universitaria reconocían que estudiarían con una presencia mayoritariamente de hombres, algunas ya lo habían vivido, lo que permitía que no fuera un obstáculo en su proceso de inserción.

"En el bachillerato estuve un semestre en la tarde y luego me cambie de grupo, cuando me cambie fue cuando me empecé a juntar casi con puros hombres, porque la mayoría del salón eran puros hombres casi y pues ahí me gradué de técnico en programación, solicite mi título en técnico". ES 4IM

"Fui a una plática al Tecnológico donde me decían que pos sí que iban a entrar puros hombres, cuando llegue a hacer el examen de admisión pues estaba yo sola de mujer, no había nadie, y dije no pos si es cierto voy a tener que ser yo sola de mujer y no ya al ratito llego otra mujer". EL 6IM

Una referencia significativa es lo que implica la nota periodística del Heraldo del 1 de junio del 2018 que dice al texto:

"Representaran a México en Emiratos Árabes Tres jimenenses, a Expo Ciencia Internacional.

Crean sistema de monitoreo de humedad a control remoto, único en el mundo, para ahorrar agua en riego de nogales.
Jiménez, Chih.- Tres Jóvenes jimenenses estudiantes del Instituto Tecnológico de Jiménez obtuvieron un pase directo al Mundial de la Expociencia internacional que realizara el próximo año en Abu Dabi, capital de los Emiratos Árabes, siendo el primer grupo integrado únicamente por mujeres que logra representar a México en un evento de esa talla, a donde acudirán potenciales inversionistas, inventores y científicos de todo el mundo.”

Menciona que tres mujeres estudiantes de sexto semestre de Ingeniería mecatrónica trabajaron por nueve meses seguidos en el desarrollo de un producto que permita el ahorro sustancial del agua de riego desde su celular, buscando convertirse en futuras empresarias.

Tres de las catorce mujeres entrevistadas son reconocidas por sus competencias STEM con un proyecto que permite coadyuvar al desarrollo sustentable.

\section{Agradecimiento}

Se agradece al TecNM/ ITCdJ en especial a sus estudiantes entrevistadas, por el tiempo y sus historias de vida compartidas y al CID.

\section{Conclusiones}

El narrar las historias de las mujeres que han decidido estudiar una carrera de ingeniería, es un hilo conductor como dice Bolivar (2001) en el cual no sólo permite el análisis y reflexión en el otro, supone una reflexibilidad sobre la vida y se explicita en la crónica del yo, normalmente a instancias de otro en la geografía social y temporal de la vida. Es poder reflexionar en las capacidades que como mujeres y su capacidad de trasformar su realidad.

Las estudiantes desarrollan competencias STEM a lo largo de la vida, fortaleciéndolas en su etapa universitaria al elegir carreras profesionales que les permiten aprender y reconstruir sus aptitudes, conocimientos $\mathrm{y}$ destrezas que les brindan como resultado un desempeño en la ingeniería.

Es trascendental reconocer que las tendencias mundiales se orienten hacia el desarrollo de la economía, basada en el conocimiento y desarrollo de tecnologías modernas de las TIC, lo que provoca un aumento en la necesidad de educación. 
Se observarán empleados y puestos que requieran mayores competencias profesionales (Ślusarczyk, 2015). Las mujeres se convierten en agentes de cambio a través de la educación, no sólo receptores pasivos de programas de empoderamiento. Ha aumentado el número de mujeres en carreras relacionadas a la tecnología lo que puede coadyuvar a superar los estereotipos sobre la división de profesiones en hombres y mujeres, y apoyar el proceso de proporcionar igualdad de oportunidades para ambos sexos en el mercado laboral (Solihati, 2019)

Las competencias STEM estarán desarrollándose en los profesionistas del hoy para un mañana que ya está aquí.

\section{Referencias}

Álvarez-Gayou, J. L. (2003). Cómo hacer investigación cualitativa. Fundamentos y metodología 2(3). México: Paidós.

Arredondo Trapero, F. G., Vázquez Parra, J. C., \& Velázquez Sánchez, L. M. (2019). STEM y brecha de género en Latinoamérica. Revista de El Colegio de San Luis, 9(18), 137-158.

Álvarez Roberto (1 junio 2018) Tres Jimenenses, a Expo Ciencias Internacional. El Heraldo de Chihuahua.

Atkinson, P. (2005, September). Qualitative research-Unity and diversity. In Forum Qualitative Sozialforschung/Forum: Qualitative Social Research (Vol. 6, No. 3).

Bolívar, A., Domíngo, J., \& Fernandez, M. (2001). La investigación biográfica-narrativa en educación. Enfoque y metodología. España: La Muralla.

Bolívar, A. (2012). Metodología de la investigación biográfico-narrativa: recogida y análisis de datos. Dimensões epistemológicas e metodológicas da pesquisa (auto) biográfica. Porto Alegre: EDIPUCRS, 79-109.

Gardner, H. (2011). La inteligencia reformulada. Las inteligencias múltiples en el siglo XXI. Cambridge: Paidós

Graña, F. (2008). El asalto de las mujeres a las carreras universitarias "masculinas": cambio y continuidad en la discriminación de género. PRAXIS educativa, 12(12), 77-86.
Huchim Aguilar, D., \& Reyes Chávez, R. (2013). La investigación biográfico-narrativa, una alternativa para el estudio de los docentes. Revista Electrónica" Actualidades Investigativas en Educación", 13(3).

Palermo, A. I. (2006). El acceso de las mujeres a la educación universitaria. Revista argentina de sociología, 4(7), 11-46.

Sánchez, B. I.; Jiménez, G, Montoya J, Barraza, C. (2016) Las inteligencias múltiples y el rendimiento en matemáticas. Revista de Aplicación Científica y Técnica, 2(3), 27-31.

Solihati, N. (2019, February). Women in Engineering (Women Choose Education in Engineering). In 5th UPI International Conference on Technical and Vocational Education and Training (ICTVET 2018). Atlantis Press.

Ślusarczyk, B., \& Broniszewska, A. (2015). Evidence from Poland on women in engineering education, Global Journal of Engineering Education, 17(1).

Tecnológico Nacional de México TecNM (2019)

https://www.tecnm.mx/informacion/sistemanacional-de-educacion-superior-tecnologica

Million Women Mentors (2015). Million Women Mentors. Advancing women and girls in STEM careers through mentoring. Recuperado de https://www.millionwomenmentors.org/ 